\title{
A REMARK CONCERNING MULTIPLICITIES
}

\author{
CRAIG HUNEKE ${ }^{1}$
}

\begin{abstract}
We prove that if a complete local ring $A$ containing a field satisfies Serre's condition $S_{n}$ and the multiplicity of $A$ is at most $n$, then $A$ must be Cohen-Macaulay.
\end{abstract}

The purpose of this paper is to prove the following theorem.

THEOREM 1.1. Let $(A, m)$ be a complete local ring containing a field. Suppose $A$ satisfies Serre's condition $S_{n}$, and let $e(A)$ be the multiplicity of $A$. If $e(A) \leq n$, then $A$ is Cohen-Macaulay.

Recall a Noetherian ring $A$ is said to satisfy $S_{n}$ if

$$
\operatorname{depth} A_{p} \geq \inf (n, \operatorname{ht}(p)) \text { for all } p \in \operatorname{Spec}(A) \text {. }
$$

Two corollaries of Theorem 1.1 are known.

COROLLARY $1.2[4]$. Let $(A, m)$ be a complete local ring containing a field which is unmixed and has multiplicity one. Then $A$ is regular.

Proof. We may assume $A / m$ is infinite. Choose a minimal reduction [5] $x_{1}, \ldots, x_{d}$ for $m$, where $d=\operatorname{dim}(A)$. Then $e(A)=e(\mathbf{x} ; A)$, the multiplicity of $A$ with respect to $\mathbf{x}=\left(x_{1}, \ldots, x_{d}\right)[\mathbf{5}$, Theorem $1, \mathrm{p}$. 46]. Since $A$ is Cohen-Macaulay by Theorem 1.1, $e(\mathbf{x} ; A)=l(A / \mathbf{x})$, the length of $A / \mathbf{x}[\mathbf{6}]$. However, since $e(A)=1$, $\mathbf{x}$ must be equal to $m$. Thus $A$ is regular.

COROLLARY 1.3 (IKEDA). Let $(A, m)$ be a complete local ring containing a field such that $A$ satisfies $S_{2}$, and $e(A)=2$. Then $A$ is a hypersurface; that is, $A=R /(f)$ where $R$ is a regular local ring and $f \neq 0$.

Proof. As in Corollary 1.2, we may assume $k=A / m$ is infinite; and $\mathbf{x}=$ $\left(x_{1}, \ldots, x_{d}\right)$ is a minimal reduction of $m$. Thus, $2=e(A)=e(\mathbf{x} ; A)=l(A / \mathbf{x})$, the latter equality following from the fact that $A$ is necessarily Cohen-Macaulay by Theorem 1.1. Therefore $l(m / \mathbf{x})=1$, and we may map $R=k\left[\left[X_{1}, \ldots, X_{d}, Y\right]\right]$ onto $A$ by sending $X_{i}$ to $x_{i}$ and $Y$ to the lifting of a generator of $m / \mathbf{x}$. Since $\operatorname{dim} A=d$, the kernel of the map from $R$ onto $A$ is a height one ideal $I$ of $R$. Since $A$ is Cohen-Macaulay, $I$ is unmixed. As $R$ is factorial $I=(f)$ is principal.

REMARK 1. Both corollaries have been shown without the assumption that $A$ contains a field, and most probably Theorem 1.1 is valid in this case. However we shall use the direct summand theorem and the syzygy theorem, which are only known to hold when $A$ contains a field.

Received by the editors November 11, 1981.

1980 Mathematics Subject Classification. Primary 13 H15.

Key words and phrases. Multiplicity, local ring, Cohen-Macaulay.

${ }^{1}$ Supported by NSF Postdoctoral Fellowship.

(C) 1982 American Mathematical Society 0002-9939/81/0000-0390/\$01.50 
REMARK 2. Nagata [4, Example 2, p. 203] gives an example of a local domain of multiplicity one which is not regular. Thus the completeness of $A$ is vital. Of course, this assumption may be removed if we assume $A$ has Cohen-Macaulay formal fibers.

ProOF OF THEOREM 1.1. As above, we may assume $k=A / m$ is infinite and choose a minimal reduction $\mathbf{x}=\left(x_{1}, \ldots, x_{d}\right)$ of $m$. Set $R=k\left[\left[x_{1}, \ldots, x_{d}\right]\right] \subseteq$ $A$. Then $A$ is a finite $R$-module, and $R$ is isomorphic to a power-series ring in $d$-variables over $k$. In particular, $R$ is regular. We have the equality $e(A)=e(\mathbf{x} ; A)$, and $e(\mathbf{x} ; A)$ is just the multiplicity $e_{R}(A)$ of $A$ as an $R$-module.

Observe that $e_{R}(A)=\operatorname{rank}_{R} A$. By definition, $\operatorname{rank}_{R} A=\operatorname{dim}_{K} K \otimes_{R} A$, where $K$ is the fraction field of $R$. This equality can be seen as follows. If $M$ is an $R$-module the degree of the Hilbert polynomial of $M$ is bounded by $\operatorname{dim}(M)$. Consequently if $0 \rightarrow N \rightarrow M \rightarrow T \rightarrow 0$ is exact and $\operatorname{dim} T<\operatorname{dim} N=\operatorname{dim} M$, then it follows that $e_{R}(N)=e_{R}(M)$. It now easily follows that if $M$ is a module of positive rank $k$ over $R$, then $e_{R}(M)=k$.

By the direct summand theorem [3, Theorem 2], the inclusion $R \subseteq A$ splits as $R$-module. Write $A=R \oplus M$. (Here we use the fact that $A$ contains a field.) Since rank $A=e(A) \leq n, \operatorname{rank} M \leq n-1$.

Recall that a module $N$ over $R$ is said to satisfy $S_{k}$ if $\operatorname{depth} N_{p} \geq \min (k$, ht $p)$ for all $p \in \operatorname{Spec}(R)$. We claim $M$ satisfies $S_{n}$. Let $p \in \operatorname{Spec}(R)$. Then $\operatorname{depth} A_{p}=$ $\min \left(\operatorname{depth} M_{p}\right.$, depth $\left.R_{p}\right)$, so that $\operatorname{depth} M_{p} \geq \operatorname{depth} A_{p}$.

As $A$ is finite over $R, \operatorname{ht}(p A)=\operatorname{ht} p$. It follows that $\operatorname{depth} A_{p} \geq \min (n, \operatorname{ht} p)$ since $A$ satisfies $S_{n}$. Thus $M$ satisfies $S_{n}$. By the theorem of Auslander and Bridger [1], $M$ is an $n$th syzygy. We may now use the recent result of Evans and Griffith [2]: if $M$ is a module of finite projective dimension, and $M$ is an $n$th syzygy, then $M$ is free provided rank $M<n$. As rank $M \leq n-1$ in our case, we conclude that $M$ (and thus $A$ ) is a free $R$-module. It follows that $A$ is Cohen-Macaulay.

REMARKS AND ACKNOWLEDGEMENT. The author would like to point out that the idea of using the direct summand conjecture is due to Ikeda in his proof of Corollary 1.2, and also would like to thank S. Goto for valuable comments. Finally we note that Hochster has shown that the direct summand conjecture implies the syzygy conjecture.

\section{REFERENCES}

1. M. Auslander and M. Bridger, Stable module theory, Mem. Amer. Math. Soc. No. 94 (1969).

2. G. Evans and P. Griffith, The syzygy problem, Ann. of Math. (2) 114 (1981), 323-333.

3. M. Hochster, Contracted ideals from integral extensions of regular rings, Nagoya Math. J. 51 (1973), 25-43.

4. M. Nagata, Local rings, Krieger, Huntington, N.Y., 1975.

5. D. G. Northcott and D. Rees, Reduction of ideals in local rings, Proc. Cambridge Philos. Soc. 50 (1954), 145-158.

6. J. P. Serre, Algèbre locale multiplicités, Lecture Notes in Math., vol. 11, Springer-Verlag, Berlin, Heidelberg and New York, 1975.

Department of Mathematics, UNiVersity of IllinoIs, URbana, Illinois 61801 